Bangladesh J. Plant Taxon. 26(2): 205-218, 2019 (December)

(C) 2019 Bangladesh Association of Plant Taxonomists

\title{
DNA BARCODING AND MORPHO-ANATOMICAL CHARACTERS OF TWO FORMS OF CONVOLVULUS ARVENSIS L. (CONVOLVULACEAE) GROWN IN ASIR REGION, SAUDI ARABIA
}

\author{
Mahmoud Moustafa*, SaAd Alamri ${ }^{1}$, Ali Shati, Mohmed Al-Kahtani, \\ Sulaiman Alrumman and AbdelraheEm TawFek ${ }^{2}$ \\ Department of Biology, College of Science, King Khalid University, 9004, Abha, \\ Kingdom of Saudi Arabia (KSA)
}

Keywords: Convolvulus arvensis $\mathrm{L}$; rbcL; matK; ITS.

\begin{abstract}
DNA barcoding and morpho-anatomical characters of two forms of Convolvulus arvensis L. growing in Asir region, Saudi Arabia were studied. We applied three molecular markers, ITS, $r b c \mathrm{~L}$ and $m a t \mathrm{~K}$ for phylogenetic reconstruction in combination with 24 morpho-anatomical characters to provide proposal for the recognition of its population under two forms. Plant samples were collected from ten various populations of $C$. arvensis supposed to be of two different forms, and finally these have been found to be of two new forms (small leaf) and form (large leaf) for the flora of Saudi Arabia. TWINSPAN results showed that there was negative group with twenty one objects including stem length, stem thickness, seed shapes, petiole length, leaf apex, leaf length, petal circumference, leaf width, capsule length, capsule width, stamen length, anther width, anther length, carpel length, venations type, pollen grains shape, T.S of stem, root and leaf, stigma shape and anther base surface and positive group including hairs, stomata and anther shape. Dendrogram upon morpho-anatomical characters resolved two main clusters, one cluster of form (large leaf) and the other of form (small leaf). Amplified gene region for the form (small leaf)-rbcL showed $100 \%$ identity with $C$. lineatus; form (small leaf)-mat $\mathrm{K}$ and form (large leaf)-mat $\mathrm{K}$ had $99 \%$ identity with Calystegia sepium (L.) R. Br. Form of (small leaf)-ITS had $100 \%$ identity with that of $C$. arvensis; form (large leaf)-rbcL had $100 \%$ identity with those of $C$. arvensis and $C$. lineatus. Form (large leaf)-ITS had 99\% identity with that of $C$. arvensis. The results of molecular phylogenetic analyses based on certain morphological and $r b c \mathrm{~L}$ data support two main clusters within $C$. arvensis which is consistent with two forms, form (small leaf) and form (large leaf).
\end{abstract}

\section{Introduction}

Convolvulus arvensis $\mathrm{L}$. is one of the most common weeds all over the world found mostly in about 54 countries distributed in 32 different economic crops (Holm et al., 1997). It was named as a field-bind weed due to its extensive deep root system together with long-term seed bank that were considered as key features to the noxious weed status (Gianoli, 2001). C. arvensis plants are native to Europe and grows extensively in Mediterranean climates, temperate and tropical regions and in many other climatic zones (Lyons, 1998). Field bindweed was considered as a perennial vine of the glorious family Convolvulaceae which spreads by rhizome or/and seed and its prostrate weak -stem, often twine forming tangled dense mats (Wiese and Phillips, 1976; Gleason and

*Corresponding author. Email: mfmostfa@kku.edu.sa.

${ }^{1}$ Prince Sultan Bin Abdulaziz Center For Environmental and Tourism Research and Studies-King Khalid University (KSA).

${ }^{2}$ Department of Botany, Faculty of Science, South Valley University, Qena, Egypt. 
Cronquist, 1963). Its underground rhizomes can extend between $5 \mathrm{~cm}$ and $2.6 \mathrm{~m}$ and deeply penetrate the lands and its climbing stems can reach up to 1.5 meter. Till now, no infraspecific category for C. arvensis has been reported in Saudi Arabia (Collenette, 1985; Migahid, 1978 and 1996) while one variety has been registered in the North America, namely C. arvensis var. obtusifolium Choisy (Robinson and Fernald 1908). Gray (1970) also opined that there were two forms of field bindweed, one had leaves with broad basal lobes and on wide cordate base named as form cardifolius Lasch. and the other one had acute ear-shaped lobes on the base of the leaves and an oblong, linear, or lanceolate blades named as form auriculatus Descr. In Europe more than sixty varieties of $C$. arvensis have been characterized and the description used to sort out among them have been attributed to environmental conditions (Kogan, 1986). In USA, it has been found that there were many intermediate characteristics to the identified varieties of $C$. arvensis, however, researchers were discouraging to apply specific name to them (Brown, 1946). The Kingdom of Saudi Arabia (KSA) contains one of the most diverse floras among the surrounding countries due to its various climatic regime such as Mediterranean, semiarid, and arid climates and cold climate in high altitude areas of $2700 \mathrm{~m}$ above sea level. These environmental conditions in KSA might affect $C$. arvensis populations to be splitted in to two or more forms, and based on this ground we contemplate to examine whether two or more forms of $C$. arvensis are growing in KSA.

In the past, the study of plant taxonomy was based to a large extent on the morphological characters of the floral parts. Recently, an application of molecular techniques in taxonomy is being increasingly used to interpret the phylogenetic relationship among plant taxa. Molecular techniques have emerged as an accurate additional tool in providing authentic and unambiguous identification of specific taxa and to study boundaries among specific species (Kaukas and Rollinson 1997).

DNA sequence data have notably helped with a high level of accuracy in reconstruction of the phylogeny of different groups of organisms presenting totally new understandings on their taxonomy and phylogeny (Friesen et al., 2006; Asmussen et al., 2006; Lefébure et al. 2006). Also, many data previously obtained from different sources other than genetic materials are being neglected.

Hence, this study was carried out to evaluate the morphological, anatomical and molecular variations in $C$. arvensis of Asir region, KSA, in order to investigate the existence of any infra specific taxon and understand the phylogenetic relationships within this species based on DNA sequence data obtained from $m a t \mathrm{~K}, r b c \mathrm{~L}$ and ITS regions.

\section{Materials and Methods \\ Morpho-anatomical character states}

Vegetative characters including stem length, stem thickness, petiole length, leaf apex, leaf length, petal circumference, leaf width, capsule length, capsule width, stamen length, anther width, anther length, carpel length, venations type, pollen grains shape, stigma shape, anther base surface, hairs, stomata, anther shape, seed shape, transverse section in stem, in root and in leaves, were studied either with the naked eye or under a stereo microscope (Leica, 10446322, 2X WD). Five populations for each of the two forms viz. form (small leaf) and form (large leaf) were selected and characteristics of five individuals from each of this population were investigated.

The measurement was considered as an averaged plus/minus maximum and minimum for various quantitative parameters. According to Stevens (1991) all described characters were quantitatively and qualitatively expressed by denoting a coding value, to prevent misrepresentation of the possible variation among the investigated plants. 


\section{Statistical analysis}

The multivariate statistical analysis for morpho-anatomical characters was carried out using Community Analysis Package (CAP) Statistics program, version 5. Cluster analysis for the morpho-anatomical characters including (Euclidean Ward's (Nath et al., 2014; Ward, 1963), TWINSPAN (Two Way Indicator Species Analysis, Manhas et al., 2009) and Jaccard's Similarity Coefficient were performed.

\section{DNA barcoding}

Genomic DNA from form (small leaf) and form (large leaf) from healthy fresh leaf were extracted using a Qiagen DNAeasy Plant Mini kit. matK forward (5'-ACCCAGTCC ATCTGGAAATCTTGGTTC-3'), and matK reverse (5'-CGTACAGT ACTTTTGTGTTTACGA G-3') and $r b c \mathrm{~L}$ forward (5'-ATGTCACCACAAACAGAGACTAAAGC-3'), $r b c \mathrm{~L}$ reverse (5'-GT AAAATCAAGTCCACCRCG-3') and ITS4 (5'-TCCTCCGCTTATTGATATGC-3') (Costion et al., 2011;Yuan et al., 2015) regions were amplified by applying Polymerase Chain Reaction (PCR) technique. $50 \mu \mathrm{L}$ reaction solutions having, $1 \times \mathrm{PCR}$ reaction buffer, $0.2 \mathrm{mM}$ deoxynucleotide triphosphate (dNTPs), $2.5 \mathrm{mM} \mathrm{MgCl}_{2}, 0.2 \mu \mathrm{M}$ each primer, $1 \mathrm{U}$ of Taq DNA polymerase and $0.75-1.5 \mu \mathrm{L}$ genomic DNA of two examined extracts for amplifications process. PCR program was adjusted as followings: $3 \mathrm{~min}$ at $94^{\circ} \mathrm{C}$ for initial denaturation, 35 cycles of 30 Sat $94^{\circ} \mathrm{C}$ for denaturation, $30 \mathrm{~S}$ at $48^{\circ} \mathrm{C} / 57^{\circ} \mathrm{C}$ for annealing, 1 min at $72^{\circ} \mathrm{C}$ for primer extension, and a final extension for $10 \mathrm{~min}$ at $72^{\circ} \mathrm{C}$. All products gained from PCR and stained with ethidium bromide were visualized on $1 \%$ agarose gel and a UV table. By using $1 \mathrm{~kb}$ DNA ladder the amplification of the primer had been confirmed and estimated. The amplified bands were introduced for sequencing by using Applied Biosystems, 3500 genetic analyser.

Resulted sequence from primer was compared with each other by using Bio edit version 7.1.11 and Clustal $\mathrm{W}$ multiple sequence alignment to investigate molecular phylogenetic analysis (Tamura et al., 2011; Grimm et al., 2006). Obtained sequences from the $m a t \mathrm{~K}, r b c \mathrm{~L}$ and ITS regions for the two taxa were compared by other relevant sequences using BLAST (Basic Local Alignment Search Tool). A split is a bipartition in a species set, that divides all dataset of species into two groups: a functional out-group and a functional in-group (Huson et al., 2010).

\section{Results and Discussion}

Morpho-anatomical features

The list of morphological and anatomical characters that were investigated together with their character states is shown in the Figs $1.1-1.5$ and Table 1. The resulted dendrogram based on these characters was resolved in two main discrete clusters of form (small leaf) and form (large leaf) for the ten populations of which were separated at linkage distance of 52.9. While five populations of form (small leaf) were further resolved in to two sub-clusters at 1.2 (Fig. 2).

The Jaccard's pairwise similarity coefficient values for ten populations of $C$. arvensis ranged from 0.0833 to 1.0, whereas those among the populations of form (small leaf) varied from 0.96 to 1.00 and of form (large leaf) didn't vary, rather remained always highest (1.00) (Table 2). These values between the populations of two forms (small leaf) and form (large leaf) ranged from 0.0833 to 0.125 . Whereas, this coefficient was 0.0833 between P2 and P4 of forms (small leaf) and P1-P5 of forms (large leaf) and it was 0.125 between P1, P3 and P5 of forms (small leaf) and P1-P5 of forms (large leaf, Table 2).

TWINSPAN output for the classification of ten populations revealed that there was negative group with twenty one characters including stem length, stem thickness, seed shapes, petiole length, leaf apex, leaf length, petal circumference, leaf width, capsule length, capsule width, 
stamen length, anther width, anther length, carpel length, venations type, pollen grains shape, transverse section (TS) in stem, in root, in leaf, stigma shape and anther base surface. Also it showed that there was positive group of three characters comprising hairs, stomata and anther shape.
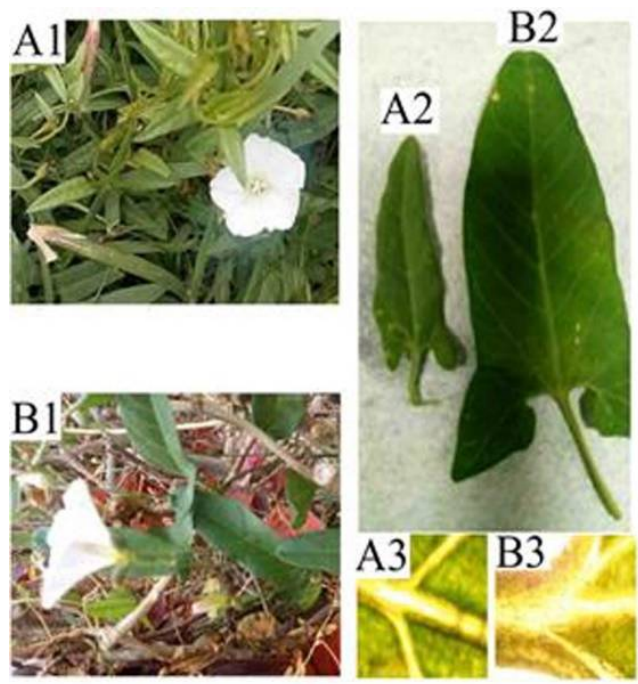

Fig.1.1. Filed images of habit (A1), leaf (A2) and venations (A3) of $C$. arvensis form small leaf and habit (B1), leaf (B2) and venations (B3) of $C$. arvensis form large leaf.
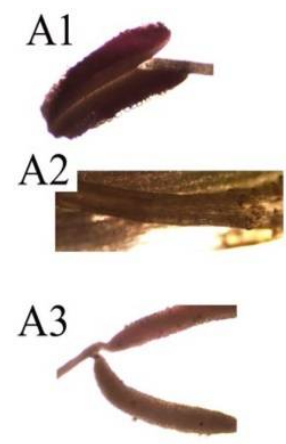

A4

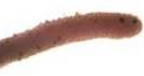

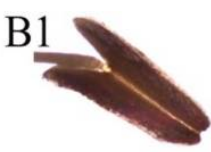

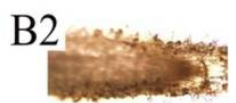

B3

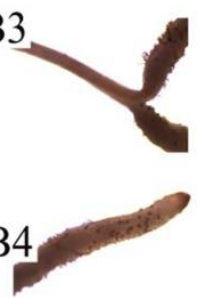

Fig.1.3. Anther (A1); anther base surface (A2); Bifid stigma (A3) and stigma rod (A4) of $C$. arvensis form small leaf and anther (B1); anther base surface (B2); bifid stigma (B3) and stigma rod (B4) of C. arvensis form large leaf.
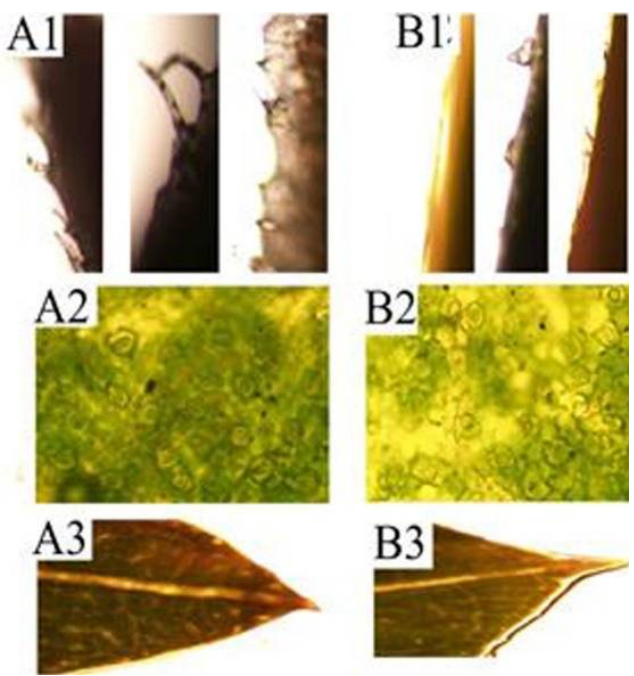

Fig.1.2. Hairs (A1), stomata (A2) and leaf apex (A3) of C. arvensis $\mathrm{L}$. form small leaf and hairs (B1), stomata (B2) and leaf apex (B3) of C. arvensis form large leaf.

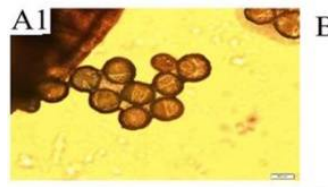

B1
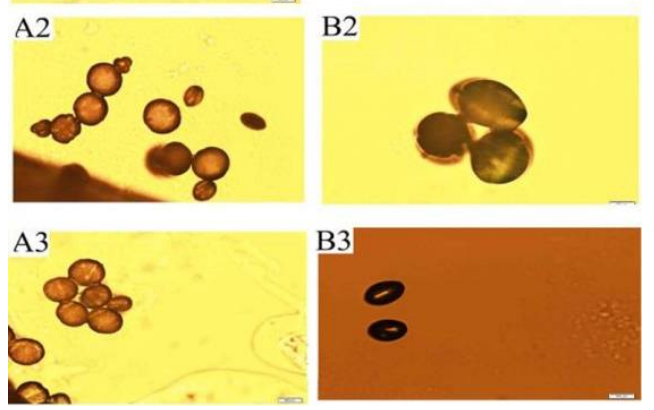

Fig. 1.4. Pollen grains (A1, $\mathrm{A} 2$ and $\mathrm{A} 3$ ) of $C$. arvensis $\mathrm{L}$. form small leaf and (B1, B2 and B3) of $C$. arvensis form large leaf. 

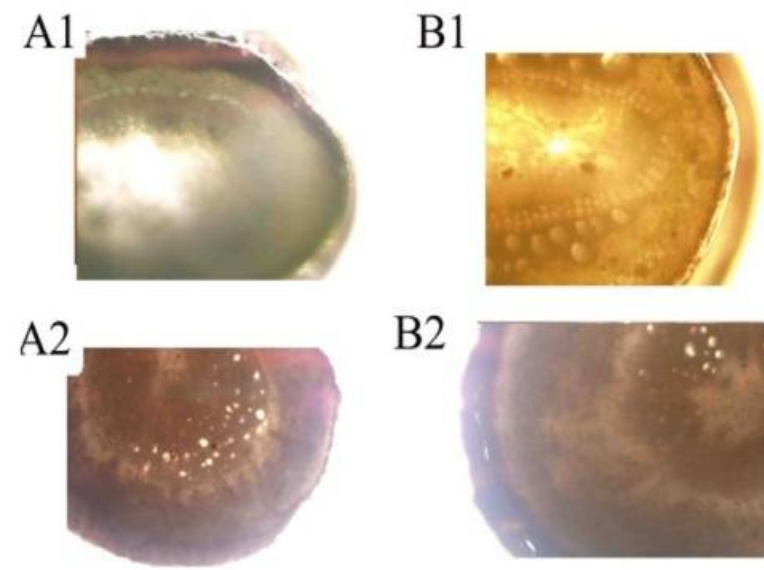

B2
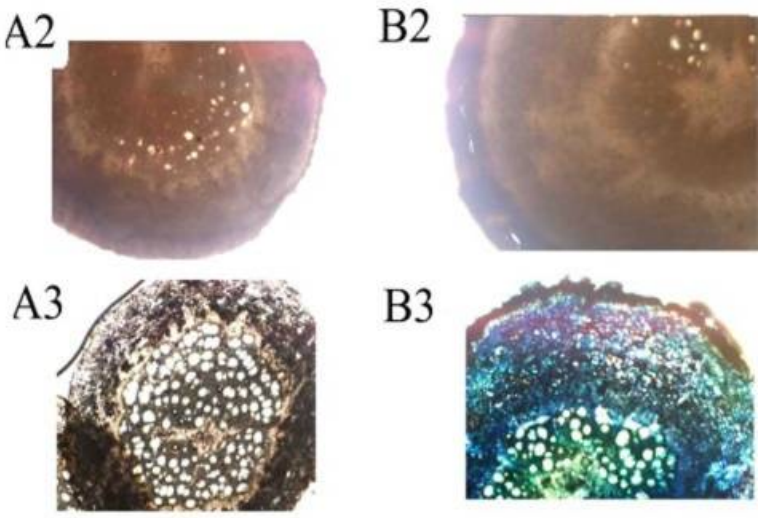

B3

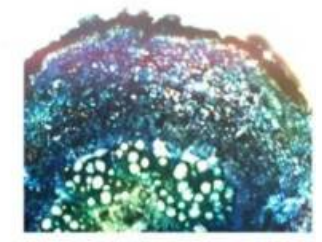

A4

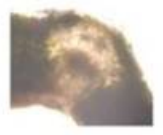

B4

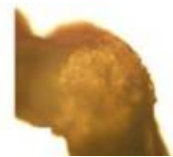

Fig.1.5. Transverse section (T.S.) of stem (A1); root (A2 and A3); leaf (A4) of C. arvensis L. form small leaf and T.S. of stem (B1); root (B2 and B3); and leaf (B4) of C. arvensis form large leaf.

As the morpho-anatomical characters of $C$. arvensis from Saudi Arabia showed distinct variation between the two forms, namely form (small leaf) and form (large leaf). Therefore, these variations can be used to clearly distinguish between some closely related subspecies, varieties, and forms.

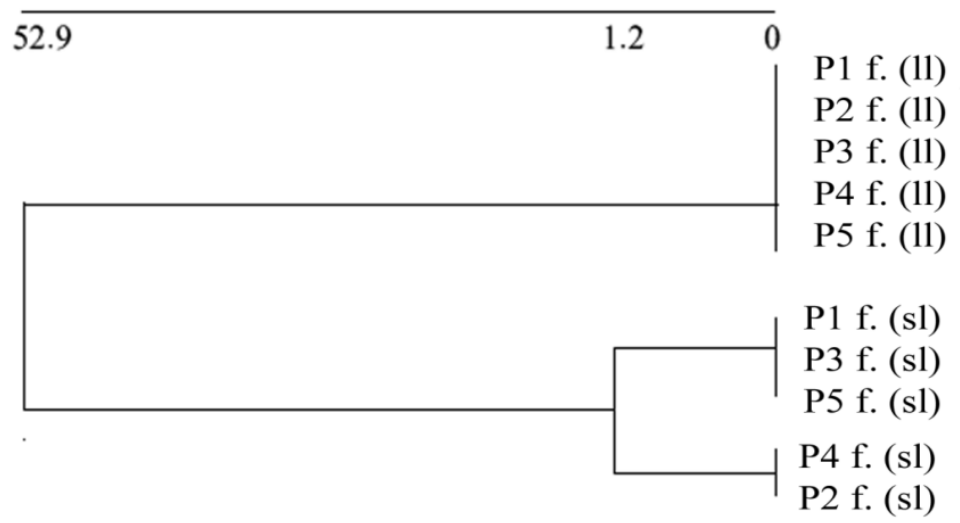

Fig. 2. Dendrogram based on morpho-anatomical characters from ten populations of $C$. arvensis form (small leaf (sl)) and form (large leaf (11)) of C. arvensis. 
Table 1. Morphological and anatomical characters for $C$. arvensis form (small leaf) and $C$. arvensis form (large leaf).

\begin{tabular}{|c|c|c|}
\hline Characters & $\begin{array}{l}\text { Characters states of } C \text {. arvensis form } \\
\text { (small leaf) from five populations }\end{array}$ & $\begin{array}{l}\text { Characters states of } C \text {. arvensis form (large } \\
\text { leaf) from five populations }\end{array}$ \\
\hline Stem length & $\begin{array}{l}99.45 \mathrm{~cm}(\operatorname{Max} 105 \mathrm{~cm} \pm \operatorname{Min} 90 \mathrm{~cm}) \\
\text { almost prostrate }\end{array}$ & $\begin{array}{l}224.57 \mathrm{~cm}(\operatorname{Max} 270 \mathrm{~cm} \pm \text { Min } 190 \mathrm{~cm}) \\
\text { climbing }\end{array}$ \\
\hline Stem thickness & $0.97 \mathrm{ml}(\mathrm{Max} 1.1 \mathrm{ml} \pm \mathrm{Min} 0.88 \mathrm{ml})$ & $1.21 \mathrm{ml}(\operatorname{Max} 1.28 \mathrm{ml} \pm \operatorname{Min} 1.15 \mathrm{ml})$ \\
\hline Seed shapes & Short (small)-3 seeds - beared shaped & Long-3 seeds - beared shaped \\
\hline Petiole length & $\begin{array}{l}\text { Shortened, } 6.7 \mathrm{ml}(\text { Max } 7.1 \mathrm{ml} \pm \text { Min } \\
6.00 \mathrm{ml})(\text { Fig 1.1.A2) }\end{array}$ & $\begin{array}{l}\text { Flattened, } 25.72 \mathrm{ml}(\text { Max } 26.0 \mathrm{ml} \pm \text { Min } \\
25.0 \mathrm{ml} \text { ) (Fig 1.1.B2) }\end{array}$ \\
\hline Leaf apex & $\begin{array}{l}\text { Acuminate on the upper side } \\
\text { (Fig.1.2. A3) }\end{array}$ & $\begin{array}{l}\text { Mucronate on the upper side } \\
\text { (Fig.1.2. B3) }\end{array}$ \\
\hline Leaf length & $\begin{array}{l}46.32 \mathrm{ml}(\operatorname{Max} 47.7 \mathrm{ml} \pm \operatorname{Min} 45 \mathrm{ml}) \\
\text { (Fig 1.1. A2) }\end{array}$ & $\begin{array}{l}\text { 111.47. } \mathrm{ml}(\text { Max } 115 \mathrm{ml} \pm \text { Min } 110 \mathrm{ml}) \\
\text { (Fig 1.1.B2) }\end{array}$ \\
\hline Petal circ. & $\begin{array}{l}\text { 64.31 ml (Max } 65.49 \mathrm{ml} \pm \text { Min } 60.92 \\
\text { ml) (Fig 1.1. A1) }\end{array}$ & $\begin{array}{l}94.33 \mathrm{ml}(\mathrm{Max} 97.03 \mathrm{ml} \pm \text { Min } 90.34 \mathrm{ml} \text { ) } \\
\text { (Fig 1.1. B1) }\end{array}$ \\
\hline Leaf width & $\begin{array}{l}15.08 \mathrm{ml}(\text { Max } 15.3 \mathrm{ml} \pm \text { Min } 14.8 \\
\mathrm{ml})(\text { Fig 1.1. A2) }\end{array}$ & $\begin{array}{l}40.40 \mathrm{ml}(\mathrm{Max} 41.2 \mathrm{ml} \pm \text { Min } 39.98 \mathrm{ml}) \\
\text { (Fig 1.1.B2) }\end{array}$ \\
\hline Capsule length & $5.58 \mathrm{ml}(\operatorname{Max} 5.9 \mathrm{ml} \pm \operatorname{Min} 5.1 \mathrm{ml})$ & $9 \mathrm{ml}(\operatorname{Max} 9.7 \mathrm{ml} \pm \operatorname{Min} 9.4 \mathrm{ml})$ \\
\hline Capsule width & $3.26 \mathrm{ml}(\mathrm{Max} 3.4 \mathrm{ml} \pm \mathrm{Min} 3.10 \mathrm{ml})$ & $5.26 \mathrm{ml}(\operatorname{Max} 5.50 \mathrm{ml} \pm \operatorname{Min} 5.10 \mathrm{ml})$ \\
\hline Stamen length & $\begin{array}{l}9.25 \mathrm{ml}(\mathrm{Max} 9.4 \mathrm{ml} \pm \operatorname{Min} 9.1 \mathrm{ml} \\
\text { (Fig.1.3. A2) }\end{array}$ & $\begin{array}{l}11.49 \mathrm{ml}(\text { Max } 13.0 \mathrm{ml} \pm \text { Min } 10.1 \mathrm{ml} \\
\text { (Fig.1.3. B2) }\end{array}$ \\
\hline Anther width & $\begin{array}{l}0.09 \mathrm{ml}(\mathrm{Max} 0.10 \mathrm{ml} \pm \text { Min } 0.08 \mathrm{ml}) \\
\text { (Fig.1.3. A1) }\end{array}$ & $\begin{array}{l}0.12 \mathrm{ml}(\mathrm{Max} 0.14 \mathrm{ml} \pm \text { Min } 0.12 \mathrm{ml}) \\
\text { (Fig.1.3. B1) }\end{array}$ \\
\hline Anther length & $\begin{array}{l}\text { 2.16 } \mathrm{ml}(\operatorname{Max} 2.22 \mathrm{ml} \pm \operatorname{Min} 2.12 \mathrm{ml}) \\
\text { (Fig.1.3. A1) }\end{array}$ & $\begin{array}{l}2.28 \mathrm{ml}(\mathrm{Max} 2.41 \mathrm{ml} \pm \operatorname{Min} 2.23 \mathrm{ml}) \\
\text { (Fig.1.3. B1) }\end{array}$ \\
\hline Carpel length & $\begin{array}{l}\text { Style: } 11.4 \mathrm{ml}(\text { Max } 11.5 \mathrm{ml} \pm \text { Min } \\
11.1 \mathrm{ml} \text {; (Fig.1.3. A3); stigma: } 3.16 \\
\text { ml (Max } 3.23 \mathrm{ml} \pm \text { Min } 3.10 \mathrm{ml}) \\
\text { (Fig.1.3. A4) }\end{array}$ & $\begin{array}{l}\text { Style: } 12.24 \mathrm{ml}(\text { Max } 12.4 \mathrm{ml} \pm \text { Min } 12.1 \\
\text { ml); (Fig.1.3. B3); stigma: } 3.26 \mathrm{ml}(\mathrm{Max} \\
3.33 \mathrm{ml} \pm \text { Min } 3.20 \mathrm{ml} \text { ) (Fig.1.3. B4) }\end{array}$ \\
\hline Venations & $\begin{array}{l}\text { From } 6 \text { to } 7 \text {; nearly even pinnate } \\
\text { (Fig.1.1. A3) }\end{array}$ & From 7 to 8; alternate (Fig.1.1. B3) \\
\hline Pollen grains & $\begin{array}{l}\text { In cluster with three grooves (Fig. } \\
\text { 1.4. A1, } 2 \text { and } 3 \text { ) }\end{array}$ & $\begin{array}{l}\text { Solitary or in triplex with prominent three } \\
\text { grooves (Fig. 1.4. B1, } 2 \text { and 3) }\end{array}$ \\
\hline Anther shape & Sagitate (Fig. 1.3. A1) & Sagitate (Fig. 1.3. B1) \\
\hline Stigma shape & Bifid (Fig. 1.3. A3) & Bifid (Fig. 1.3. B3) \\
\hline $\begin{array}{l}\text { Anther base } \\
\text { surface }\end{array}$ & Cylindrical (Fig. 1.3. A2) & Cylindrical (Fig. 1.3. B2) \\
\hline Hairs & $\begin{array}{l}\text { Many types mainly compounds } \\
\text { (Fig.1.2. A1) }\end{array}$ & $\begin{array}{l}\text { Many types mainly compounds (Fig.1.2. } \\
\text { B1) }\end{array}$ \\
\hline Stomata & $\begin{array}{l}\text { Kidneys shapes of stomata (Fig.1.2. } \\
\text { A2) }\end{array}$ & Kidneys shapes of stomata (Fig.1.2. B2) \\
\hline T.s in Stem & Fig.1.5. A1 & Fig.1.5. B1 \\
\hline T.S. in Root & Fig.1.5. A2 and A3 & Fig.1.5. B2 and B3 \\
\hline T.S. in leaf & Fig.1.5. A4 & Fig.1.5. B4 \\
\hline
\end{tabular}


Table 2. Jaccard's similarity coefficient among 24 characters of two forms (small leaf (sl)) and (large leaf (ll)) of $C$. arvensis.

\begin{tabular}{llllll}
\hline & \multicolumn{1}{c}{$\begin{array}{c}\text { P1. } \\
\text { form (sl) }\end{array}$} & $\begin{array}{c}\text { P2. } \\
\text { form (sl) }\end{array}$ & $\begin{array}{c}\text { P3. } \\
\text { form (sl) }\end{array}$ & $\begin{array}{c}\text { P4. } \\
\text { form (sl) }\end{array}$ & $\begin{array}{c}\text { P5. } \\
\text { form (sl) }\end{array}$ \\
\hline P1. form (sl) & 1.0000 & & & & \\
P2. form (sl) & 0.9583 & 1.0000 & & & \\
P3. form (sl) & 1.0000 & 0.9583 & 1.0000 & & \\
P4. form (sl) & 0.9583 & 1.0000 & 0.9583 & 1.0000 & \\
P5. form (sl) & 1.0000 & 0.9583 & 1.0000 & 0.9583 & 1.000 \\
P1. form (ll) & 0.125 & 0.08333 & 0.125 & 0.08333 & 0.125 \\
P2. form (ll) & 0.125 & 0.08333 & 0.125 & 0.08333 & 0.125 \\
P3. form (ll) & 0.125 & 0.08333 & 0.125 & 0.08333 & 0.125 \\
P4. form (ll) & 0.125 & 0.08333 & 0.125 & 0.08333 & 0.125 \\
P5. form (ll) & 0.125 & 0.08333 & 0.125 & 0.08333 & 0.125 \\
\hline
\end{tabular}

Table 2. (Contd.) Jaccard's similarity coefficient among 24 characters of two forms (small leaf (sl)) and form (large leaf (ll)) of $C$. arvensis.

\begin{tabular}{lccccc}
\hline & $\begin{array}{c}\text { P. } \\
\text { form (1l) }\end{array}$ & $\begin{array}{c}\text { P2. } \\
\text { form (1l) }\end{array}$ & $\begin{array}{c}\text { P. } \\
\text { form (1l) }\end{array}$ & $\begin{array}{c}\text { P4. } \\
\text { form (ll) }\end{array}$ & $\begin{array}{c}\text { P5. } \\
\text { form (ll) }\end{array}$ \\
\hline P1. form (sl) & & & & & \\
P2. form (sl) & & & & & \\
P3. form (sl) & & & & \\
P4. form (sl) & & & & \\
P5. form (sl) & & & & \\
P1. form (ll) & 1.0000 & 1.0000 & & & \\
P2. form (ll) & 1.0000 & 1.0000 & 1.0000 & & \\
P3. form (ll) & 1.0000 & 1.0000 & 1.0000 & 1.0000 & \\
P4. form (ll) & 1.0000 & 1.0000 & 1.0000 & 1.0000 & 1.0000 \\
P5. form (ll) & 1.0000 & & & & \\
\hline
\end{tabular}

This conclusion is consistent with Sa`ad (1967) who distinguished between the morphologically very similar species $C$. valentinusis and $C$. supinus on the light of the minor variations in their leaf shapes and the presence or absence of hairs on both sides of their leaves .

DNA sequence analysis

The resulted sequence length of the $m a t \mathrm{~K}, r b c \mathrm{~L}$ and ITS regions for the two forms of $C$. arvensis were in between 577-862 bp for form (large leaf) $-r b c \mathrm{~L}$ and form (small leaf)-matK respectively (Table 3). The GC content ranged between 33.874 for form (small leaf)-mat $\mathrm{K}$ to 56.086 for form (small leaf)-ITS. Form (small leaf)-rbcL showed $100 \%$ identity with C. lineatus (KT178135.1) and C. arvensis (KM360729.1), (100\%). Form (small leaf)-matK and form (large leaf)-matK had 99\% identity with C. arvensis (KT176616.1), C. arvensis (MG946999.1), Calystegia sepium (JN894556.1) and Calystegia sepium (FJ395438.1). Form (small leaf)-ITS had $100 \%$ identity with $C$. arvensis (AY560274.1), C. arvensis (AY558826.1), C. arvensis 
(KC528905.1), C. arvensis (KJ021876.1) and C. arvensis (JQ062475.1). Form (large leaf)-rbcL had $100 \%$ identity with $C$. arvensis (KT178135.1), C. lineatus (MF158799.1) and C. arvensis (KM360729.1). Form (large leaf)-ITS had 99\% with C. arvensis (AY560274.1), C. arvensis (AY558826.1), C. arvensis (KC528905.1), C. arvensis (KJ021876.1), and C. arvensis (JQ062475.1).

Table 3. Read Length, GC Content and BlastN Report of $r b c \mathrm{~L}$, matK and ITS regions of form (small leaf) and form (large leaf).

\begin{tabular}{|c|c|c|c|}
\hline Form name & $\begin{array}{l}\text { Read } \\
\text { Length }\end{array}$ & GC Content & $\begin{array}{l}\text { BlastN Report (Description -Accession no. and } \\
\text { Identities Pct. }(\%)\end{array}$ \\
\hline $\begin{array}{l}\text { form (small leaf)- } \\
r b c L\end{array}$ & 582 & 45.70446735395189 & $\begin{array}{l}\text { C. arvensis (KT178135.1), (99\%) } \\
\text { C. arvensis (MG946887.1), (99\%) } \\
\text { C. lineatus (KT178135.1), (100\%) } \\
\text { C. arvensis (KM360729.1), (100\%) } \\
\text { C. arvensis (AY100993.1), (99\%) }\end{array}$ \\
\hline $\begin{array}{l}\text { form (small leaf)- } \\
\operatorname{mat} \mathrm{K}\end{array}$ & 862 & 33.874709976798144 & $\begin{array}{l}\text { C. arvensis (KT176616.1), (99\%) } \\
\text { C. arvensis (MG946999.1), (99\%) } \\
\text { Calystegia sepium (JN894556.1), (99\%) } \\
\text { C. cneorum (HQ384565.1), (98\%) } \\
\text { Calystegia sepium (FJ395438.1), (99\%) }\end{array}$ \\
\hline $\begin{array}{l}\text { form (small leaf)- } \\
\text { ITS }\end{array}$ & 690 & 56.086956521739125 & $\begin{array}{l}\text { C. arvensis (AY560274.1), (100\%) } \\
\text { C. arvensis (AY558826.1), (100\%) } \\
\text { C. arvensis (KC528905.1), (100\%) } \\
\text { C. arvensis (KJ021876.1), (100\%) } \\
\text { C. arvensis (JQ062475.1), (100\%) }\end{array}$ \\
\hline $\begin{array}{l}\text { form (large leaf)- } \\
r b c \mathrm{~L}\end{array}$ & 577 & 45.407279029462735 & $\begin{array}{l}\text { C. arvensis (KT178135.1), (100\%) } \\
\text { C. lineatus (MF158799.1), (100\%) } \\
\text { C. arvensis (MG946887.1), (99\%) } \\
\text { C. arvensis (KM360729.1), (100\%) } \\
\text { C. arvensis (AY100993.1), (99\%) }\end{array}$ \\
\hline $\begin{array}{l}\text { form (large leaf)- } \\
m a t \mathrm{~K}\end{array}$ & 856 & 33.8785046728972 & $\begin{array}{l}\text { C. arvensis (KT176616.1), (99\%) } \\
\text { C. arvensis (MG946999.1), (99\%) } \\
\text { Calystegia sepium (JN894556.1), (99\%) } \\
\text { C. cneorum (HQ384565.1), (98\%) } \\
\text { Calystegia sepium (FJ395438.1), (99\%) }\end{array}$ \\
\hline $\begin{array}{l}\text { form (large leaf)- } \\
\text { ITS }\end{array}$ & 680 & 55.88235294117647 & $\begin{array}{l}\text { C. arvensis (AY560274.1), (99\%) } \\
\text { C. arvensis (AY558826.1), (99\%) } \\
\text { C. arvensis (KC528905.1), (99\%) } \\
\text { C. arvensis (KJ021876.1), (99\%) } \\
\text { C. arvensis (JQ062475.1), (99\%) }\end{array}$ \\
\hline
\end{tabular}

The output of identity matrix sequences resulted from $r b c \mathrm{~L}, m a t \mathrm{~K}$ and ITS are shown (Figure 3 and Table 4).The sequence identity matrix revealed that the highest identity matrix ranged between 100\% among form (large leaf)-ITS, form (small leaf)-rbcL and form (small leaf)- $r b c \mathrm{~L}$, 
followed by $99.3 \%$ among form (large leaf)-matK and form (small leaf)-mat $\mathrm{K}$. The next highest sequence identity matrix $97.8 \%$ was found among form (large leaf)-ITS, form (small leaf)-rbcL and form (small leaf)-ITS. The lowest sequence identity matrix $26.3 \%$ was recorded among form (small leaf)-ITS and form (large leaf)-rbcL (Table 4).

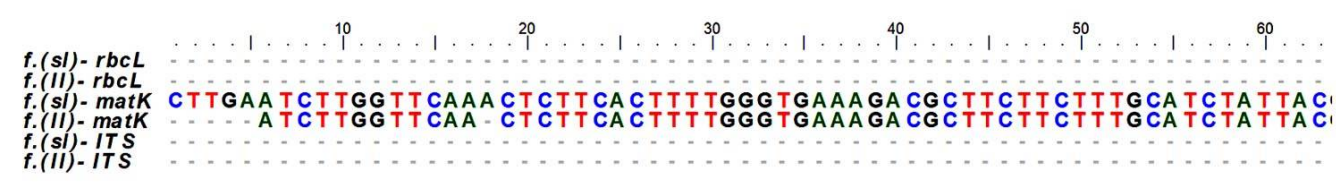

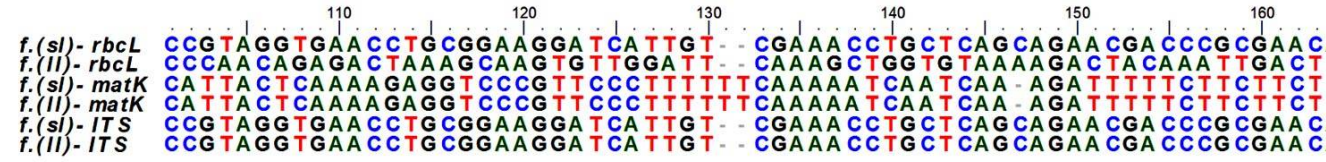

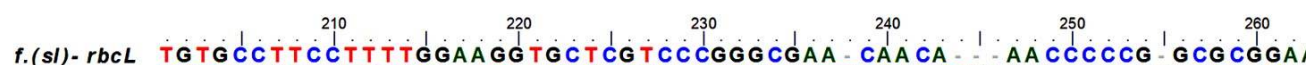

f.(s): rbCL TGTTCCTTCCT TTGGAAGG TGCTCG TCC CGGCGAA CAACA AACCCCCG GCGCGGAA

f. (s)-matK TTTTTCTTTCTCCG TAA CCAATCTCTTCA TT TACGAT. CAACC.. AGTTTTGGAGCCCTTT

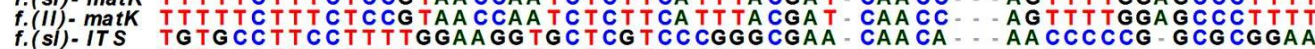

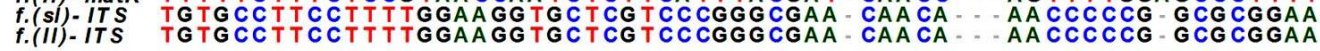

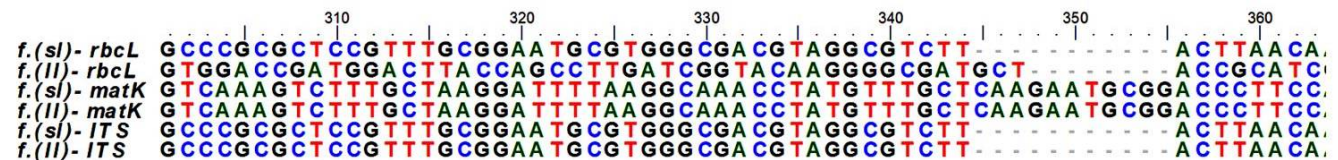

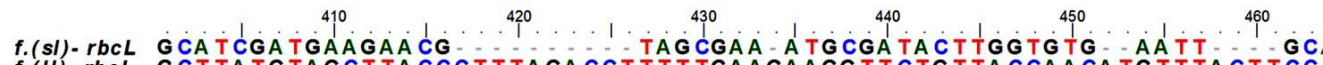
f. (II)- $r b C L$ GCT TA TGTAGCTTACCCTTTAGACCTTTTTGAAGAAGG TC TGTTACCAACA TGTTTACTTCC f.(s)-matK GCTTCAA-AAGGAA CGCTTCTTT TGA TGT CGAA A AGGAACTA TTA TTTTG TTAA TT TTTGGC f.(s):IIS GCA CGA AGAAGAAACG.

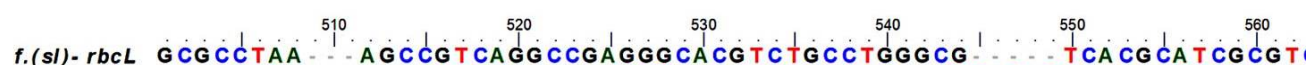
f.(II). $r b c L$ GCGCCTCTA f.(s))-matK CAA TA TAAGCCAACTATCCAGCCA TTCCCCTTGACTTTA TGGGCTA TCTTTCAAG TA TTCCACT

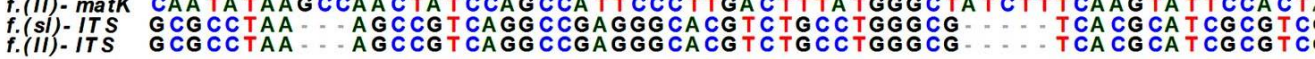

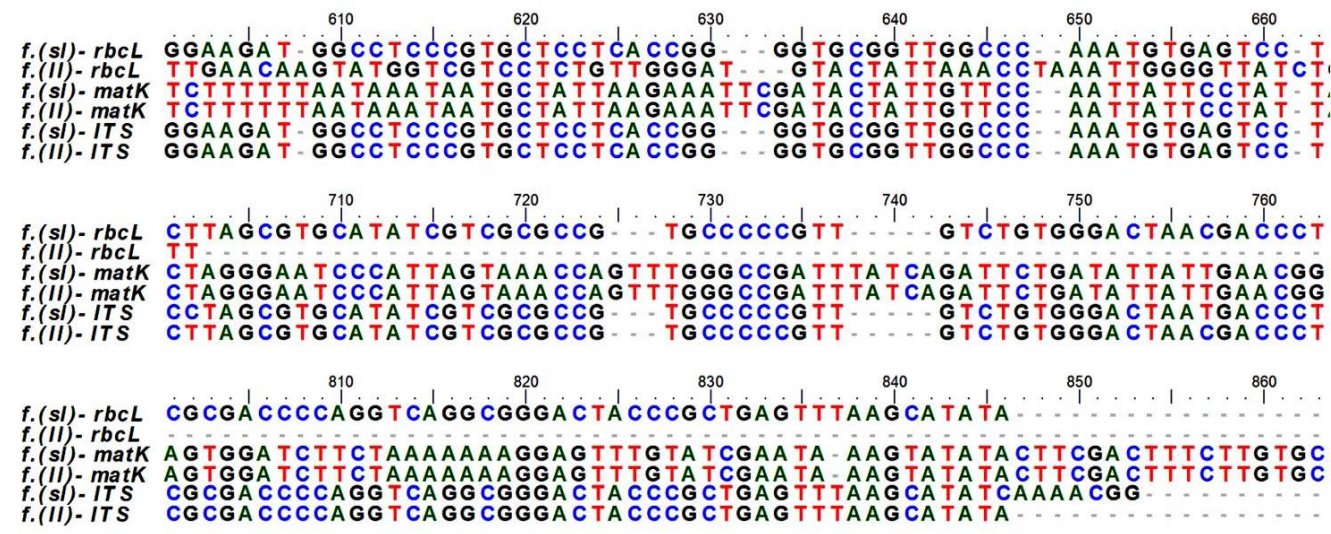

Fig. 3. Sequence alignment for $r b c \mathrm{~L}$, matK and ITS region forms (small leaf (sl)) and form (large leaf (ll)) of C. arvensis. 
Table 4. Sequence identity matrix for $r b c \mathrm{~L}$, matK and ITS genes region of form (small leaf(sl)) and form (large leaf (ll)) of C. arvensis.

\begin{tabular}{|c|c|c|c|c|c|c|}
\hline & $\begin{array}{l}\text { form }(\mathrm{sl})- \\
\quad r b c \mathrm{~L}\end{array}$ & $\begin{array}{l}\text { form (ll) } \\
-r b c \mathrm{~L}\end{array}$ & $\begin{array}{c}\text { Form (sl) } \\
-m a t \mathrm{~K}\end{array}$ & $\begin{array}{c}\text { form (ll) } \\
\text {-matK }\end{array}$ & $\begin{array}{c}\text { Form }(\mathrm{sl}) \\
\text {-ITS }\end{array}$ & $\begin{array}{c}\text { form (11) } \\
\text {-ITS }\end{array}$ \\
\hline form $(\mathrm{sl})-r b c \mathrm{~L}$ & ID & 0.263 & 0.352 & 0.355 & 0.978 & 1.000 \\
\hline form (1l) $-r b c \mathrm{~L}$ & 0.263 & ID & 0.283 & 0.285 & 0.258 & 0.263 \\
\hline form $(\mathrm{sl})-m a t \mathrm{~K}$ & 0.352 & 0.283 & ID & 0.993 & 0.354 & 0.352 \\
\hline form (ll) - $m a t \mathrm{~K}$ & 0.355 & 0.285 & 0.993 & ID & 0.356 & 0.355 \\
\hline form (sl) -ITS & 0.978 & 0.258 & 0.354 & 0.356 & ID & 0.978 \\
\hline form (ll) -ITS & 1.000 & 0.263 & 0.352 & 0.355 & 0.978 & ID \\
\hline
\end{tabular}

The maximum likelihood (ML) analysis was based on $r b c \mathrm{~L}, \operatorname{mat} \mathrm{K}$ and ITS regions of two forms of $C$. arvensis (Figure 4). The ML tree was resolved in to two clusters of the two forms. Cluster 1 formed at the branch length of 0.8 was consisted of form (small leaf)- $r b c \mathrm{~L}+$ ITS and form (large leaf)-ITS. Cluster 2 at branch length of 0.3 was consisted of the clade of form (large leaf)-mat $\mathrm{K}$ and form (small leaf)-matK. The branch length of the out-group form (large leaf)-rbcL was 0.8 .

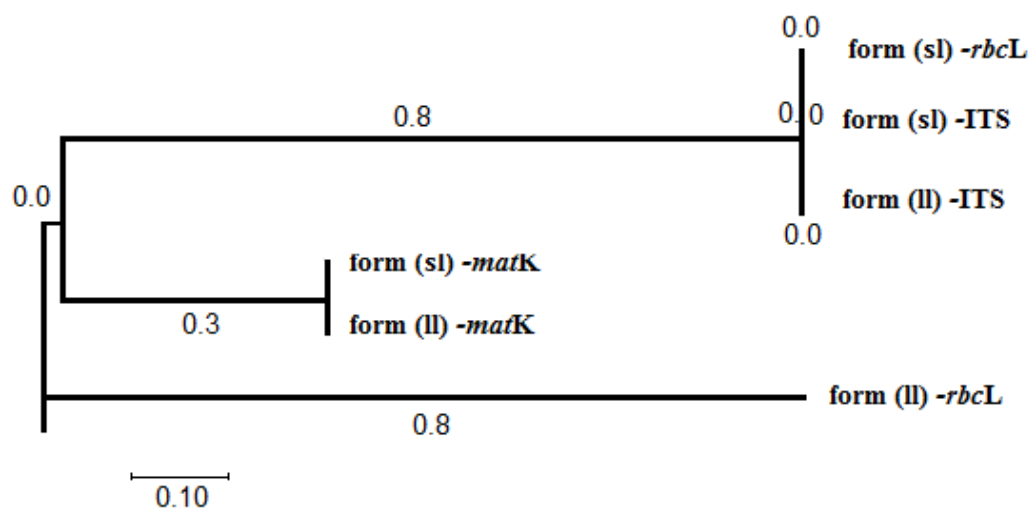

Fig. 4. ML tree generated through maximum likelihood analysis of $r b c \mathrm{~L}$, mat $\mathrm{K}$ and ITS sequence data of two forms (small leaf (sl)) and form (large leaf (ll)) of C. arvensis.

The nucleotide composition resulted from $r b c \mathrm{~L}$, matK and ITS regions of two forms of $C$. arvensis are shown in (Table 5). The average of all the three nucleotide sequences had a total of 724 positions, in the final data set, for Pos \#1 (241), for Pos \#2 and for Pos \#3 (240). It revealed that form (small leaf)- $r b c \mathrm{~L}$ had the highest Guanine $(\mathrm{G})$ content $(28.7 \%$ ) and lowest Adenine (A) (20.6\%), while form (large leaf)-rbcL had the highest Thymine (T) and Adenine contents of $27.4 \%$ and $27.2 \%$ respectively and lowest Cytosine (C) content of 22.0. Form (small leaf)-matK and form (large leaf)-matK had the highest Thymine $(37.7 \%)$ and lowest Guanine (15.9\%). Form (small leaf)-ITS and form (large leaf)-ITS had high amount of Guanine (287\%) and low amount of Adenine (20.6\%). The degree of bias showed avraiation depends upon the codon composition. At the first codon position for form (small leaf) $r b c \mathrm{~L}$, the usage of $\mathrm{G}$ was $33.3 \%$, and those of the other bases were $25.4 \%$ (C), $19.3 \%$ (A) and $22.0 \%(\mathrm{~T})$. At the second codon position, the content 
of $\mathrm{T}$ was $23.00 \%$ and those of the other bases were $28.6 \%(\mathrm{C}), 22.00 \%(\mathrm{~A})$ and $26.4 \%(\mathrm{G})$. At the third codon position, the base usage was $\mathrm{T}(26.00 \%)$, C (27.6\%), A (20.4\%) and G $(26.2 \%)$ (Shrivastava et al., 2013). The detailed numbers of codon composition for form (large leaf)-rbcL, form (small leaf)-matK, form (large leaf)-matK, form (small leaf)-ITS, form (large leaf)- ITS are shown in Table 5.

Table 5. Nucleotide composition of $r b c \mathrm{~L}$, mat K and ITS regions of form (small leaf (sl)) and form (large leaf (II)) of C. arvensis.

\begin{tabular}{lllllllllll}
\hline Source & $T(U)$ & $C$ & $A$ & $G$ & Total & $T-1$ & $C-1$ & $A-1$ & $G-1$ & Pos \#1 \\
\hline form (sl)-rbcL & 23.5 & 27.2 & 20.6 & 28.7 & 680.0 & 22 & 25.4 & 19.3 & 33.3 & 228.0 \\
form (ll)-rbcL & 27.4 & 22.0 & 27.2 & 23.4 & 577.0 & 34 & 20.9 & 27.2 & 18.3 & 191.0 \\
form (sl)-matK & 37.7 & 18.0 & 28.4 & 15.9 & 862.0 & 37 & 15.3 & 29.6 & 18.1 & 287.0 \\
form (ll)-matK & 37.7 & 18.0 & 28.4 & 15.9 & 856.0 & 37 & 15.1 & 29.8 & 17.9 & 285.0 \\
form(sl)-ITS & 23.2 & 27.2 & 20.7 & 28.8 & 690.0 & 22 & 25.0 & 19.8 & 33.2 & 232.0 \\
form (ll)- ITS & 23.5 & 27.2 & 20.6 & 28.7 & 680.0 & 22 & 25.4 & 19.3 & 33.3 & 228.0 \\
Avg. & 29.6 & 22.9 & 24.6 & 22.9 & 724.2 & 29 & 20.7 & 24.5 & 25.3 & 241.8 \\
& & & & & & & & & & \\
\hline
\end{tabular}

Table 5. (Contd.) Nucleotide composition of $r b c L$, matK and ITS regions of form (small leaf (sl)) and form (large leaf (li)).

\begin{tabular}{lllllllllll}
\hline Source & $T-2$ & $C-2$ & $A-2$ & $G-2$ & Pos \#2 & $T-3$ & $C-3$ & $A-3$ & $G-3$ & Pos\#3 \\
\hline form (sl)-rbcL & 23 & 28.6 & 22.0 & 26.4 & 227.0 & 26 & 27.6 & 20.4 & 26.2 & 225.0 \\
form (ll)- rbcL & 21 & 18.7 & 25.9 & 34.2 & 193.0 & 27 & 26.4 & 28.5 & 17.6 & 193.0 \\
form (sl)-matK & 36 & 17.7 & 27.1 & 18.8 & 288.0 & 40 & 20.9 & 28.6 & 10.8 & 287.0 \\
form (ll)- matK & 36 & 17.8 & 26.9 & 18.9 & 286.0 & 40 & 21.1 & 28.4 & 10.9 & 285.0 \\
form (sl)- ITS & 23 & 28.7 & 22.2 & 26.5 & 230.0 & 25 & 28.1 & 20.2 & 26.8 & 228.0 \\
form (ll)- ITS & 23 & 28.6 & 22.0 & 26.4 & 227.0 & 26 & 27.6 & 20.4 & 26.2 & 225.0 \\
Avg. & 28 & 23.0 & 24.5 & 24.5 & 241.8 & 31 & 24.9 & 24.7 & 19.1 & 240.5 \\
\hline
\end{tabular}

Phylogenetic relationships based on molecular and morphological properties have been considered in many taxa (Bernardi and Crane, 2005; Ward et al., 2005). Also, combinations between DNA sequencing and morphological characters including leaf anatomy and macromorphology had been conducted to investigate the generic and subgeneric relations (Soh and Parnell, 2011). Interestingly in this work, we found concordant results from the analyses of molecular data and morphological and anatomical characters.

We found variations in morphological and anatomical characters accompanied with nucleotide sequences of two forms of $C$. arvensis. Such variations may be due to the differences in the GC percentage, nucleotide length, nucleotide bases differences, and number of gaps in each nucleotide region (Singh et al., 2016). These differences in each form of $C$. arvensis can be mainly ascribed to the varying numbers of repeat, alignment gaps, deletions, copy number sand base 
substitutions and/or additions. Base additions and substitutions are featured by very high $\mathrm{C}$ content that might form pure poly C structures (Shrivastava et al., 2013). Therefore, all of these factors individually or altogether will lead to a consequence of mutations in the lineage of $C$. arvensis forms according the prevailing environmental conditions. Variation in the amount of GC content and other nucleotides $\mathrm{A}, \mathrm{T}$ and $\mathrm{C}$ in the first, second and third position is same to that of some other plant groups, for example, Triticeae tribe (Bieniek et al., 2015) and Pisum sativum varieties (Moustafa et al., 2019).

Comparison the obtained sequences from $r b c \mathrm{~L}$, mat $\mathrm{K}$ and ITS regions with the sequences in the GenBank gave various similarity percentage with other species of convolvulus plants. For example, form (small leaf)- $r b c \mathrm{~L}$ and form (large leaf)- $r b c \mathrm{~L}$ gave a $100 \%$ similarity with $C$. lineatus and $C$. arvensis. There was no $100 \%$ similarity was obtained from form (small leaf)$m a t \mathrm{~K}$, and form (large leaf)-matK, though form (small leaf)-ITS gave $100 \%$ similarity with $C$. arvensis. Also, the molecular phylogenetic analyses showed that form (large leaf) $-r b c \mathrm{~L}$ clustered alone than other nucleotide sequences generated from matK and ITS regions. Therefore, $r b c \mathrm{~L}$ can be applied to address the problems of plant phylogeny in lower taxa, even at the level of form, since it could separate form (large leaf) from form (small leaf) and in concomitant with morphoanatomical characters of these two forms. However Baldwin et al. (1995), found that ITS could reconstruct phylogenetic relationships precisely among plant species. Li et al. (2011) found that ITS effectively resolved the problems of species by sorting out 6,286 samples from 1,757 of seed plant species. An application of ITS was also questioned. The sequencing and amplification rate of ITS primer was relatively low (i.e. $71.00 \%$ and $86.20 \%$ respectively) which might be as a result from some second-level structures on it (Huang et al., 2015; DeSalle, 2007; Waugh, 2007). Additionally, Xing et al. (2018) found that $r b c \mathrm{~L}$ and $m a t \mathrm{~K}$ were not appropriated for characterization the plant for tropical forest at the species level.

In conclusion, our results have established two different forms of $C$. arvensis (small leaf) and (large leaf) based on DNA barcoding and morpho-anatomical characters in Saudi Arabia. In addition, our results conclude that $r b c \mathrm{~L}$ sequences are suitable for characterization of $C$. arvensis at the form level.

\section{Acknowledgement}

The authors extend their appreciation to the Deanship of Scientific Research at King Khalid University for funding this work under a research project (R.G.P.77 /40).

\section{References}

Asmussen, C.B., Dransfield, J., Deickmann, V., Barfod, A.S., Pintaud, J-C. and Baker, W.J. 2006. A new subfamily classification of the palm family (Arecaceae): evidence from Plastid DNA. Bot. J. Linn. Soc. 151: $15-38$.

Baldwin, B.G, Sanderson, M.J., Porter, J.M., Wojciechowski, M.F., Campbell, C.S. and Donoghue, M.J. 1995. The ITS Region of Nuclear Ribosomal DNA: A Valuable Source of Evidence on Angiosperm Phylogeny. Ann. Mo. Bot. Gard. 182: 247-277.

Bernardi, G. and Crane, N.L. 2005. Molecular phylogeny of the humbug damshelfishes inferred from mtDNA sequences. J. Fish Biol. 54: 1210-1217.

Bieniek, W., Mizianty, M., Szklarczyk, M. 2015. Sequence variation at the three chloroplast loci (matK, rbcL, trnH-psbA) in the Triticeae tribe (Poaceae): comments on the relationships and utility in DNA barcoding of selected species. Plant Syst. Evol. 301:1275-1286

Brown, E.O. 1946. Notes on some variations in field bindweed (Convolvulus arvensis L.). Iowa State Coll. Jou. Sci. 20: 269-276. 
Collenette, S. 1985. An illustrated guide to the flowers of Saudi Arabia. - London. UK: Scorpion Publishing Press.

Costion, C., Ford, A., Cross, H., Crayn, D., Harrington, M. and Lowe, A. 2011. Plant DNA barcodes can accurately estimate species richness in poorly known floras. PLoS One. 6(11): e26841.

DeSalle, R. 2007. Phenetic and DNA taxonomy; a comment on Waugh. Bioessays 29: 1289-1290.

Friesen, N., FrITSch, R.M. and Blattner, F. 2006. Phylogeny and new intrageneric classification of Allium L. (Alliaceae) based on nuclear ribosomal DNA ITS sequences. Aliso. 22: 372-395.

Gleason, H.A. and Cronquist, A. 1963. Manual of the Vascular Plants of the Northeastern United States and Adjacent Canada. D. VanNostrand Company, Inc., Princeton, NJ.

Gianoli, E. 2001. habit in Convolvulus arvensis (Convolvulaceae). Int. J. Plant. Sci. 162(6): 1247-52.

Gray, A. 1970. Gray's Manual of Botany; a handbook of the flowering plants and ferns of central and northeastern United States and adjacent Canada, $8^{\text {th }}$ ed. D. VanNostrand Co., New York.

Grimm, G.W., Renner, S.S., Stamatakis, A. and Hemleben, V. 2006. A nuclear ribosomal DNA phylogeny of Acer inferred with maximum likelihood, splits graphs, and motif analysis of 606 sequences. Evol. Bioinform. Online 2: 1-16.

Holm, L.G., Plucknett, D, Pancho, J.V. and Herberger, J.P. 1997. The world's worst weeds: distribution and biology. The University Press of Hawaii, Honolulu.1991: 98-104.

Huang, X., Ci, X., John, G. and Li, J. 2015. Application of DNA Barcodes in Asian Tropical Trees - A Case Study from Xishuangbanna Nature Reserve, Southwest China. PLoS ONE 10: e0129295.

Huson, D.H., Rupp, R. and Scornavacca, C. 2010. Phylogenetic Net-works. Concepts, Algorithms and Applications. Cambridge University Press, Cambridge.

Kaukas, A. and Rollinson, D. 1997. Interspecific variation within the hyper variable region of the $18 \mathrm{~S}$ ribosomal RNA gene among species of Schistosoma weinland, 1858 (Digenea). Syst. Parasitol. 36: 157160.

Kogan, M. 1986. Eco-physiology and control of Convolvulus arvensis L. in Ecology and Control of Perennial Weeds in Latin America. Food and Agriculture Organization of the United Nations, Rome.

Lefébure, T., Douady, C.J., Gouy, M. and Gibert J. 2006. Relationship between morphological taxonomy and molecular divergence within Crustacea: proposal of a molecular threshold to help species delimitation. Mol. Phylogenet. Evol. 40: 435-447.

Li, D.Z., Gao, L.M., Li, H.T., Wang, H., Ge, X.J., Liu, J.Q., Chen, Z.D., Zhou, S.L., Chen, S.L., Yang, J.B., Fu, C.X., Zeng, C.X., Yan, H.F., Zhu, Y.J., Sun, Y.S., Chen, S.Y., Zhao, L., Wang, K., Yang, T. and Duan, G.W. 2011. Comparative analysis of a large dataset indicates that internal transcribed spacer (ITS) should be incorporated into the core barcode for seed plants. Proc Natl Acad Sci U S A. 108: 19641-19646.

Lyons, K.E. 1998. Element stewardship abstract for Convolvulus arvensis L. In:Field bindweed. Arlington: The Nature Conservancy, p. 1-21.

Manhas R.K., Gautam, M.K. and Kumari, D. 2009. Two Way Indicator Species Analysis (TWINSPAN) of the Herbaceous Vegetation in an Inland Wetland Ecosystem of Doon Valley Himalaya, India. J Welt. Ecol. 3: 23-31

Migahid, A. M. 1978. Flora of Saudi Arabia. King Saud University Press, Riyadh

Migahid, A.M. 1996. Flora of Saudi Arabia, Vol. 1, $4^{\text {th }}$ ed. Riyadh, Saudi Arabia: Riyadh University Press.

Moustafa, M., Alamri, S., Taha, T., Shati, A., Alrumman, S. and Al-Kahtani M. 2019. Cloning and characterization of $66 \mathrm{kDa}$ Streptavidin-Binding Peptides (SBP) of Pisum sativum L. embryo specific to var. Alaska. BIOCELL 43(3): 155-166.

Nath, U. K., Santona Rani, Paul M.R., Alam M.N., and Horneburg B. 2014. "Selection of Superior Lentil (Lens esculenta M.) Genotypes by Assessing Character Association and Genetic Diversity,”. Sci. World. J. 2014: 1-6.

Robinson, B.L. and Fernald, M.L. 1908. Gray's New Manual of Botany. 7th ed. American Book Co., New York. 
Sa'ad, F. 1967. The Convolvulus species of the Canary Islands, the Mediterranean region and the Near and Middle East. Medelingen van het Botanisch Museum en Herbarium van de Rijksuniversiteitte Utrecht, 281: $1-288$.

Shrivastava, R.R., Agrawal N. and Upadhyay M.K. 2013. Molecular Analysis, Based on 28s rDNA, of Dactylogyroides Species, Parasitizing Puntius Species. J. Biosci. Bioeng. 1(3): 25-30.

Singh, R., Ming, R. and Yu, Q. 2016. Comparative Analysis of GC Content Variations in Plant Genomes. Tropical Plant Biol. 9: 136.

Stevens, P.F. 1991. Character states, morphological variation, and phylogenetic analysis: a review. Syst. Bot. 16: $553-583$.

Tamura, K., Peterson, D., Peterson, N., Stecher, G., Nei, M. and Kumar, S. 2011."MEGA5: molecular evolutionary genetics analysis using maximum likelihood, evolutionary distance, and maximum parsimony methods." Mol. Biol. Evol. 28: 2731-9.

Ward, J. 1963. "Hierarchical grouping to optimize an objective function,”. J. Am. Stat. Assoc. 58: 236-244.

Ward, R.D., Zemlak, T.S., Innes, B.H., Last, P.R. and Hebert, P.D.N. 2005. DNA Barcoding Australia's fish species. Philos. Trans. R. Soc. B. Biol. Sci. 360: 1847-1857.

Waugh, J. 2007. DNA barcoding in animal species: progress, potential and pitfalls. Bioessays 29: 188-197.

Wiese, A. F. and Phillips, W.M. 1976. Field bindweed. Weeds Today. 7: 22-23.

Xing, B.P., Lin, H.S., Zhang, Z.L., Wang, C.G., Wang, Y.G. and Wang, J.J. 2018. DNA barcoding for identification of fish species in the Taiwan Strait. PLoS ONE 13(6): 1-13.

Yuan, Q., Zhang, B., Jiang, D., Wang, N.H., Chiou, S.J. and Huang, LQ. 2015. Identification of species and materiamedica within Angelica L. (Umbelliferae) based on phylogeny inferred from DNA barcodes. Mol. Ecol. Resour. 15(2): 358-371.

(Manuscript received on 4 June, 2019; revised on 8 December, 2019) 